\title{
A Kelch Repeat Protein, Cokel1p, Associates with Microtubules and Is Involved in Appressorium Development in Colletotrichum orbiculare
}

\author{
Ayumu Sakaguchi, Toshihiko Miyaji, Gento Tsuji, and Yasuyuki Kubo \\ Laboratory of Plant Pathology, Graduate School of Life and Environmental Sciences, Kyoto Prefectural University, \\ Kyoto 606-8522, Japan
}

Submitted 9 June 2009. Accepted 10 August 2009.

\begin{abstract}
Kelch repeat proteins are conserved in diverse organisms and some are known to mediate fundamental cellular functions. We isolated the gene CoKEL1, encoding a novel kelch repeat protein, from Colletotrichum orbiculare. Analysis of a cokel1 mutant indicated that CoKEL1 is involved in proper appressorium development and cell wall synthesis. Appressoria produced by cokell disruption mutants showed irregular shape and impairment of turgor generation and the mutant appressoria rarely penetrated to form infection hyphae in host epidermal cells. Accordingly, cokell mutants had reduced pathogenicity on host leaves compared with the wild type. Furthermore, the cokel1 mutant was more sensitive to cell-wall-degrading enzymes and showed altered labeling with the cell wall stain Calcofluor white. Cokel1p was localized on cortical and spindle microtubules in vegetative hyphae. These results suggest that Cokel1p is a microtubule-associated protein involved in infection-related morphogenesis and pathogenicity. This is the first report that a kelch repeat protein is required for the pathogenicity of a fungal plant pathogen.
\end{abstract}

Colletotrichum orbiculare (syn. C. lagenarium) is the causal agent of cucumber anthracnose disease. A series of changes in fungal morphology, including formation of a specialized infection structure called the appressorium, is important for successful infection (Kubo and Furusawa 1991). A number of metabolic functions and signal transduction pathways required for appressorium development have been characterized. CMK1, a homolog of the Saccharomyces cerevisiae FUS3/KSS1 mitogen-activated protein (MAP) kinase, and $M A F 1$, a homolog of the $S$. cerevisiae SLT2 MAP kinase, are both involved in appressorium development and pathogenicity of $C$. orbiculare (Kojima et al. 2002; Takano et al. 2000). Cstl, homologous to the STE12 transcription factor, is required for penetration peg formation by appressoria (Tsuji et al. 2003b).The cyclic AMP (cAMP) signaling pathway is also essential for pathogenicity in this fungus. The adenylate cyclase gene, $C A C 1$, and the protein kinase A (PKA) gene, $C P K 1$, were shown to be involved in conidial germination, appressorial penetration, and invasive growth into host tissue (Yamauchi et al. 2004). Metabolic pathways essential for appressorium function include melanin

Corresponding author: Yasuyuki Kubo; E-mail: y_kubo@kpu.ac.jp.

* The $\boldsymbol{e}$-Xtra logo stands for "electronic extra" and indicates that four supplemental figures are published online. Also, Figures 1 through 5 and 7 appear in color online. synthesis (Kubo et al.1996; Perpetua et al. 1996; Takano et al. 1995; Tsuji et al. 2000) and peroxisome metabolism (Asakura et al. 2009; Kimura et al. 2001).

Previously, we identified a gene, CoKEL2 (previously named ClaKEL2), encoding a kelch repeat protein, which is homologous to Schizosaccharomyces pombe tea1p. Cokel2p localizes to the apices of growing hyphae and germ tubes in a microtubule-dependent manner and is involved in normal appressorium development on artificial substrata (Sakaguchi et al. 2008). Although CoKEL2 is involved in appressorium development, the cokel 2 mutant could form normal appressoria with full virulence on the host plant when supported by calciumdependent bypass pathways which transduce plant-derived signals (Sakaguchi et al. 2008). Kelch repeat proteins are composed of multiple kelch motifs, each consisting of a segment of 44 to 56 amino acids. Several kelch repeat proteins have been functionally characterized in diverse organisms, including viruses, plants, fungi, and mammals (Adams et al. 2000). In yeast, $S$. pombe tea1p localizes to cell ends in a microtubuledependent manner and functions as part of the "polarisome" complex in establishing cell polarity (Feierbach et al. 2004; Mata and Nurse 1997). The mechanism by which tea1p is transferred to the cell apex requires binding of tealp to the partner protein tip1p at the growing microtubule plus end. In addition, the protein tea3p functions as a cell-end marker dependent on microtubules and tea1p (Arellano et al. 2002). In Aspergillus nidulans, the teal homologue TeaA was shown to be involved in polarized growth (Takeshita et al. 2008).

The relationship between kelch repeat proteins and signal transduction pathways has also been analyzed. In $S$. pombe, a mutant of the kelch repeat encoding gene RAL2 lacks mating activity and the ability to respond to mating pheromone (Fukui et al. 1989). In Saccharomyces cerevisiae, overexpression of the KEL1 gene suppresses the cell fusion defect caused by hyperactive Pkc1p, indicating that Kellp acts upstream of or parallel to the PKC1 pathway (Philips and Herskowitz 1998). More direct interactions between kelch repeat proteins and the signal transduction pathway were indicated by the report that S. cerevisiae Krh1p and Krh2p (also called Gpb1p and Gpb2p) are binding partners of the $\mathrm{G} \alpha$ protein $\mathrm{Gpa} 2 \mathrm{p}$, which functions in glucose- and sucrose-induced cAMP signaling (Harashima and Heitman 2002). The two kelch repeat proteins Krh1p and $\mathrm{Krh} 2 \mathrm{p}$ act as downstream effectors of Gpa2p and negatively regulate the PKA pathway (Peeters et al. 2007).

In this study, we identified the gene CoKELl encoding a novel kelch repeat protein in $C$. orbiculare. Targeted gene disruption indicated that CoKEL1 is required for normal appressorium development and cell wall synthesis and, in con- 
trast to CoKEL2, is essential for invasion of host tissue. Moreover, we show that Cokel1p is localized on cortical and spindle microtubules, indicating that Cokel1p is a microtubule-associated protein. Our study is the first to show that a kelch repeat protein is required for the pathogenicity of a plant-pathogenic fungus.

\section{RESULTS}

\section{Isolation of the CoKEL1 gene encoding a kelch repeat protein.}

CoKEL1 was identified as part of a screen for restriction enzyme-mediated integration (REMI) mutants of $C$. orbiculare with impaired pathogenicity. Mutant P24 was found to produce less severe lesions on cucumber cotyledons than the wild-type strain 104-T (Fig. 1A). DNA segments adjacent to the inserted plasmid were isolated from mutant $\mathrm{P} 24$ by thermal asymmetric interlaced-polymerase chain reaction (TAIL-PCR) (Fig. 1B). The TAIL-PCR product was sequenced and, on this basis, a primer pair was designed to isolate a genomic clone from a cosmid library of $C$. orbiculare. Sequence analysis revealed that the TAIL-PCR product encoded a reading frame with significant homology to proteins containing the kelch motif. The CoKEL1 locus is shown schematically in Figure 1B. The transcriptional initiation site was found by $5^{\prime}$ rapid amplification
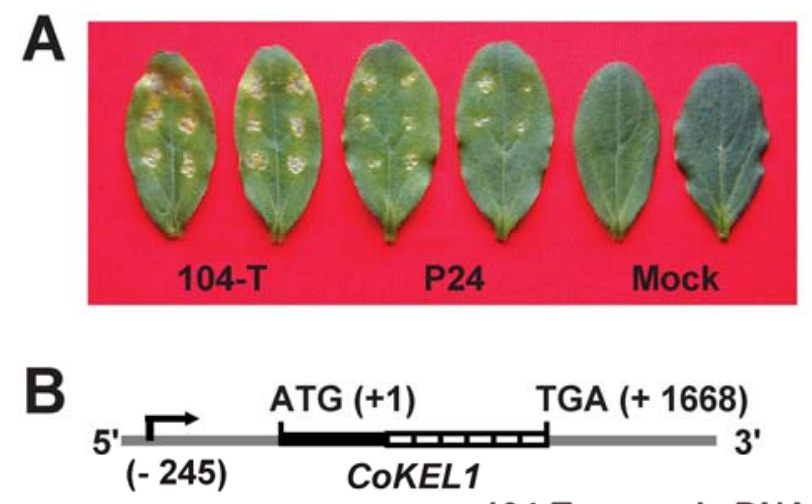
104-T genomic DNA

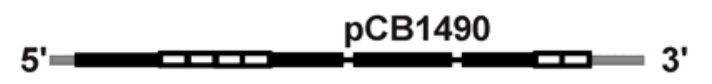

\section{P24 genomic DNA}

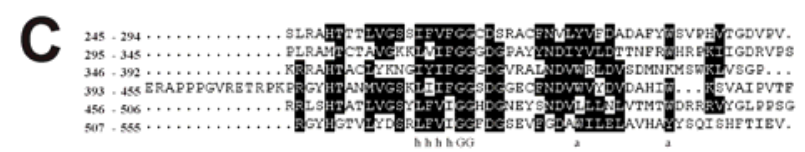

Fig. 1. Identification of the CoKEL1 gene in Colletotrichum orbiculare. A, Pathogenicity assays of restriction enzyme-mediated integration (REMI) mutant P24. Detached cucumber cotyledons were droplet inoculated with conidial suspension and incubated at $24^{\circ} \mathrm{C}$ for 5 days. 104-T, the wild type; P24, REMI mutant; Mock, distilled water control. B, Schematic representation of the CoKEL1 locus in the C. orbiculare wild-type strain and mutant P24. Exons are indicated as black boxes. The predicted kelchrepeat-encoding regions are presented as open boxes. The transcription initiation site is shown by a bent arrow. The open reading frame of CoKEL1 is shown with the predicted ATG start codon designated as +1 , and ending with a TGA termination codon at position 1668. C, Alignment of the predict kelch repeats in Cokel1p. Identical or similar residues are shown on shaded backgrounds. The lefthand numbers show amino acid positions of the kelch motifs in Cokel1p. Each amino acid sequence were examined manually for the presence of eight key conserved residues, including four hydrophobic residues (h) followed by a double glycine element (GG), and separated by two characteristically spaced aromatic residues (a) (Adams et al. 2000). of cDNA ends (RACE). The ATG codon 265 bp downstream of the transcriptional initiation site is the probable translational initiation site of CoKEL1. The upstream region of the predicted CoKEL1 open reading frame (ORF) contained upstream open reading frames (uORF) of $30 \mathrm{bp}$. Based on the preferred translation initiation codon, CoKEL1 was predicted to encode a protein of 555 amino acids, and six kelch motifs were found in the carboxyl-terminal region of Cokel1p (Fig. 1C).

To investigate the distribution of CoKELI homologs in other fungal genomes, the National Center for Biotechnology Information nonredundant protein database was searched using blastp. The deduced amino acid sequence of CoKEL1 showed high similarity to a number of fungal hypothetical proteins. A phylogenetic tree based on the amino acid sequences of CoKEL1 and CoKEL2 homologs from other fungi was constructed using MEGA version 4.0 software (Tamura et al. 2007) (Supplementary Fig. S1). Genes homologous to CoKEL1 are well conserved among ascomycetes fungi and CoKEL1 and CoKEL2 were found to belong to different clades. Cokel2p showed high homology to $S$. pombe tealp $(E$ value $=2 \mathrm{e}-77)$ but Cokel1p was less similar to tea1p $(E$ value $=2 \mathrm{e}-21)$.

\section{CoKEL1 is involved in appressorium development.}

To further define the function of CoKEL1, we used Agrobacterium tumefaciens-mediated transformation (AtMT) to generate cokell targeted disruption mutants by homologous recombination. The A. tumefaciens binary gene replacement cosmid vector p24cosKEL1Tn was introduced into the wildtype strain 104-T by AtMT. Gene replacement of CoKEL1 were verified by DNA gel blot analysis (Supplementary Fig. S2). Impairment of CoKEL1 transcription was confirmed by reverse-transcription PCR (RT-PCR). The expected fragment could be amplified from the wild-type strain and ectopic transformant but no transcript was detected in the cokell mutants.

The cokell mutants did not differ from the wild type in their growth rate on potato dextrose agar (PDA) medium when incubated at $24^{\circ} \mathrm{C}$ but showed reduced conidiation (Table 1). The wild type usually produced orange conidial masses in the central region of the colony on PDA. In contrast, cokell mutants produced black conidial masses on PDA. Conidia of cokell mutants germinated normally but formed morphologically aberrant appressoria on glass microscope slides. Approximately $90 \%$ of the appressoria produced by the wild type had a spherical form whereas approximately $50 \%$ of the appressoria produced by cokell mutants were irregular or elliptical in shape, sometimes with a swollen neck region adjoining the spore. Many mutant appressoria also showed germination from an appressorium which was not observed for the wild type

Table 1. Growth and conidiation of Colletotrichum orbiculare cokel1 mutants grown on potato dextrose agar (PDA) medium

\begin{tabular}{lcc}
\hline Strain $^{\mathbf{a}}$ & $\begin{array}{c}\text { Vegetative growth } \\
(\mathbf{m m} / \mathbf{3} \text { days })^{\mathbf{b}}\end{array}$ & $\begin{array}{c}\text { Conidia } \\
\left(\times \mathbf{1 0}^{\mathbf{7}} / \mathbf{c o l o n y}\right)^{\mathbf{c}}\end{array}$ \\
\hline 104-T & $7.77 \pm 0.26$ & $3.68 \pm 0.44$ \\
RK1-1 & $7.77 \pm 0.17$ & $0.99 \pm 0.35$ \\
RK1-2 & $7.88 \pm 0.11$ & $0.98 \pm 0.29$ \\
EK1-1 & $7.79 \pm 0.19$ & $3.08 \pm 0.80$ \\
RK1C-1 & $7.81 \pm 0.10$ & $3.71 \pm 0.53$ \\
\hline
\end{tabular}

a Strains: 104-T, wild type; RK1-1 and RK1-2, cokel1 mutant strains; EK1-1, CoKEL1 ectopic transformant; RK1C-1, GFP::CoKEL1 complemented transformant of RK1-1.

${ }^{\mathrm{b}}$ Each strain was grown on PDA at $24^{\circ} \mathrm{C}$, and radial growth was estimated by comparing colony diameters at 3 and 6 days. Radial growth was measured for five colonies of each strain, and the means and standard deviations were calculated.

${ }^{c}$ Production of conidia was measured in 7-day-old cultures. Conidia produced by five colonies of each strain were counted, and the means and standard deviations were calculated. 
(Fig. 2A and B). The generation of appressorial turgor pressure is required for mechanical penetration of the plant surface (Bechinger et al. 1999). Therefore, we evaluated the appressorial turgor of the cokell mutant using a cytorrhysis assay (Howard et al. 1991). The proportion of collapsed appressoria at glycerol concentrations above $2 \mathrm{M}$ was higher in the cokell mutant than the wild type (Fig. 2C), indicating that the mutant was unable to produce wild-type levels of turgor pressure.

\section{CoKEL1 is involved}

\section{in appressorium-mediated plant invasion.}

To assess the requirement of CoKEL1 for fungal pathogenicity, conidial suspensions were inoculated onto cucumber cotyledons. On intact cotyledons, cokell mutants produced smaller lesions than those produced by the 104-T wild-type strain (Fig. $3 \mathrm{~A})$. In contrast, when conidial suspensions were placed onto wound sites produced by scratching with a toothpick, cokell
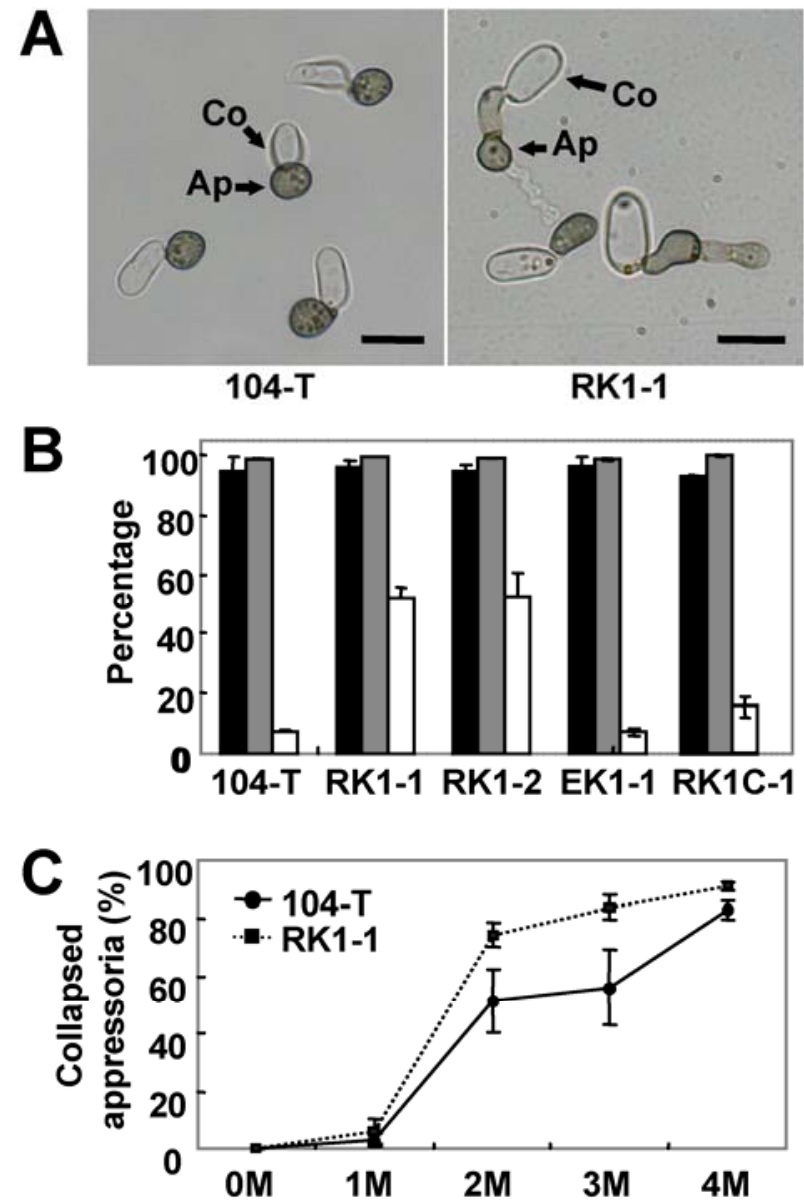

Fig. 2. Appressorium formation by cokell mutants. Conidial suspensions of each strain prepared in distilled water were incubated on multi-well glass slides at $24^{\circ} \mathrm{C}$ for $24 \mathrm{~h}$. A, Appressorium formation of cokell mutant RK1-1 and the wild-type strain 104-T. Ap, Appressoria. Co, Conidia. Scale bars $=10 \mu \mathrm{m}$. B, Percentage of conidial germination (closed bars), appressorium formation (shaded bars), and abnormal appressorium formation (open bars). 104-T, the wild type; RK1-1 and RK1-2, cokel1 mutants; EK1-1, ectopic transformant; RK1C-1, GFP::CoKEL1-1-complemented transformant of RK1-1. Approximately 200 conidia were observed per well, and three replicates were examined. Means and standard errors were calculated from three independent experiments. C, Appressorial cytorrhysis assay. Appressoria formed on multi-well glass slides were exposed to glycerol solutions ranging from 0 to $4 \mathrm{M}$ and the percentage of collapsed spherical appressoria was counted. Approximately 200 conidia were observed per well and three replicates were examined. Means and standard errors were calculated from four independent experiments. Solid line, the wild-type 104-T; dotted line, cokell mutant. mutants formed lesions indistinguishable from those of the wild type (Fig. 3B).

These results suggested that the cokell mutant has a defect in appressorium-mediated penetration of host tissues. To determine whether appressoria of the cokell mutant retained the ability to penetrate and form infection hyphae on artificial substrata, we incubated conidia of the mutant on cellophane membranes. Both the cokell mutant and the wild-type formed melanized appressoria which penetrated the cellophane membranes, producing infection hyphae inside the membrane (Fig. $4 \mathrm{~A})$. When the membranes were stained with $\mathrm{ZnCl}_{2} / \mathrm{KI} / \mathrm{I}_{2}$ solution, haloes were observed around appressoria, indicating that the cokell mutant secretes cellulose-degrading enzymes during infection (Fig 4B). Appressoria of the cokell mutants also penetrated cellophane with frequency similar to that of the wild type (Fig. 4C). To verify whether appressoria of cokell mutants penetrated cucumber epidermis, we inoculated conidial suspensions onto the abaxial surface of cucumber cotyledons, and infection sites were observed with microscopy after 3 days. In contrast to the penetration assay on cellophane membranes, appressoria of the cokell mutants rarely penetrated cucumber epidermal cells to form intracellular infection hyphae (Fig. 4A). In the wild type, 27\% of appressoria successfully penetrated to form infection hyphae on cucumber cotyledon, whereas only $3 \%$ of the cokell appressoria formed infection hyphae (Fig. 4C). On cucumber cotyledons where host resistance had been impaired by heat treatment, $58 \%$ of wild-type appressoria formed infection hyphae compared with only $7 \%$ of cokell appressoria (Fig. 4C).

\section{Cell wall synthesis is affected in the cokell mutant.}

Vegetative growth of the cokell mutant was affected at $28^{\circ} \mathrm{C}$. The colony morphology of the mutant was similar to the wild-type when incubated at $24^{\circ} \mathrm{C}$ but, at $28^{\circ} \mathrm{C}$, cokell colonies showed irregular margins (Supplementary Fig. S3). Radial growth of the wild type was also faster than the cokell mutant at $28^{\circ} \mathrm{C}$. However, the colony morphology and growth rate of the cokell mutant at $28^{\circ} \mathrm{C}$ were restored when incubated on PDA containing $0.5 \mathrm{M}$ sorbitol. Thus, hyphal growth of the cokell mutant was impaired by high temperature and restored

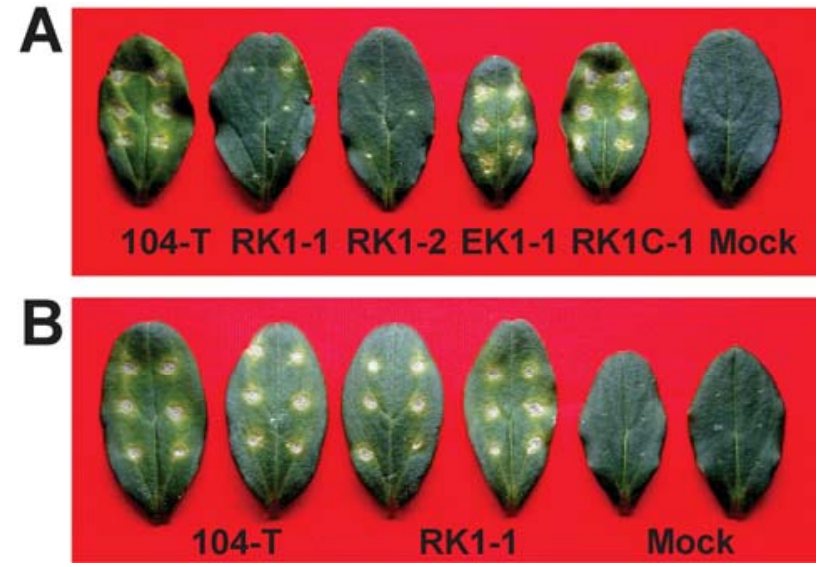

Fig. 3. Pathogenicity of cokell mutants on cucumber. A, Pathogenicity assay of cokel1 mutants on intact cucumber cotyledons. Conidial suspensions were droplet inoculated onto detached cucumber cotyledon and incubated at $24^{\circ} \mathrm{C}$ for 6 days. WT, wild-type 104-T; RK1-1 and RK1-2, cokel1 mutants; EK1-1, ectopic transformant; RK1C-1, GFP::CoKEL1-1-complemented transformant of RK1-1; Mock, distilled water control. B, Pathogenicity assay of cokell mutants on wounded cucumber cotyledons. Conidial suspensions were droplet inoculated onto wound sites (prepared by scratching the leaf surface with a sterile toothpick) and incubated at $24^{\circ} \mathrm{C}$ for 6 days. WT, wild type 104-T; RK1-1, cokel1 mutant; Mock, distilled water control. 
by high osmotic conditions. S. cerevisiae mutants of the Pkc1 and Slt2 MAP kinases, which are involved in cell wall integrity, show osmoremedial cell lysis at $37^{\circ} \mathrm{C}$ (Lee et al. 1993). Similarly, in Magnaporthe oryzae, the mycelial autolysis phenotype of mutants defective in MPS1, a MAP kinase gene closely related to Slt2, could be complemented by $1 \mathrm{M}$ sorbitol (Xu et al. 1998). Therefore, we postulated that cell wall synthesis might be impaired in the cokell mutant. To determine
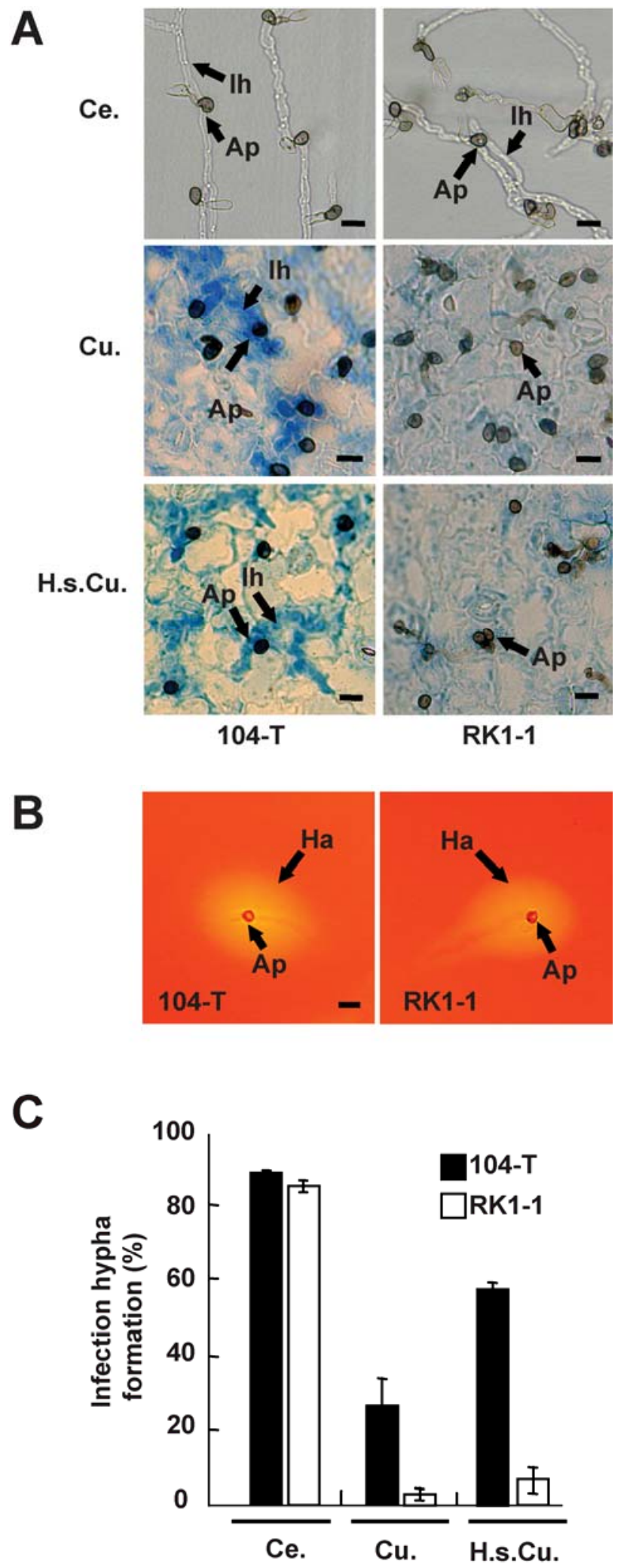

whether cokell mutants have weakened cell walls, we counted the number of protoplasts released after digestion of mycelia with lysing enzymes for 2 or $3 \mathrm{~h}$. The mean number of protoplasts released from mycelia of the cokell mutant was three times higher than from wild-type mycelia (Fig. 5A). Furthermore, the cokell mutant was more sensitive to caffeine, a chemical that affects cell wall integrity. Hyphal growth rates of the cokell mutant were approximately $85 \%$ of those of the wild type and CoKEL1-reintroduced transformant on the PDA containing $5 \mathrm{mM}$ caffeine, while remaining the same as those on PDA without caffeine (Supplementary Fig. S4). Moreover, the abundance of cell wall polysaccharides labeled by Calcofluor white was also affected in the cokell mutants. Thus, appressorial cell walls of the wild type stained strongly with Calcofluor white, whereas fluorescent labeling was not detectable in appressoria of the cokell mutant (Fig. 5B).

\section{Cokel1p is a microtubule-associated protein.}

To further elucidate the cellular role of Cokel1p, the green fluorescent protein $(G F P)$ gene was fused to the $\mathrm{C}$-terminus of CoKEL1 and expressed under the native CoKEL1 promoter. Introduction of the GFP-CoKEL1 fusion gene into the cokell mutant fully restored wild-type conidiation, appressorium formation, and pathogenicity (Figs. 2 and 3), showing that the GFP-CoKEL1 fusion protein was functional. However, GFP fluorescence could not be detected in the complemented transformants. Therefore, we constructed a GFP-CoKEL1 fusion gene expressed under a constitutive promoter. This construct was introduced into the cokell mutant and one transformant was named TFK1G. The GFP signals in some TFK1G hyphae were detected as filamentous structures similar to a cortical microtubule (data not shown). Therefore, to visualize microtubules, we introduced an $m R F P 1-\alpha T U B 1$ fusion gene into the TFK1G transformant, named TFK1GR. In most TFK1GR hyphae, GFP-Cokel1p distributed uniformly through the cytoplasm and did not show localization at specific intracellular sites whereas mRFP1- $\alpha$ TUB1 detected filamentous structures (Fig. $6 \mathrm{~A})$.

On the other hand, GFP-Cokel1p signal also corresponded to a microtubule labeled by mRFP1- $\alpha$ TUB1. In some TFK1GR hyphae, cytoplasm and filamentous structures were labeled by GFP-Cokellp and these filamentous structures colocalized with mRFP1- $\alpha$ TUB1 on cortical microtubules (Fig. $6 \mathrm{~B}$ a through $\mathrm{c}$ ). Furthermore, punctuate GFP signals were observed when the hyphae of this strain were treated with the microtubule depolymerizing drug benomyl (Fig. 6B d through f). These results indicate that Cokellp is only partly localized on microtubules.

Furthermore, the localization of GFP-Cokellp was cell cycle dependent. Thus, in cells undergoing mitosis, the long, filamentous microtubules labeled by mRFP1- $\alpha$ TUB1 disappeared and thick bundles were observed corresponding to mitotic spindles.

Fig. 4. Penetration ability of the cokell mutant on cellophane and cucumber cotyledons. A, Microscopic observation of the infection process. Conidial suspensions of the wild-type 104-T and cokell mutant were incubated on cellophane membranes at $24^{\circ} \mathrm{C}$ for $48 \mathrm{~h}$ or on the abaxial surface of cucumber cotyledons at $24^{\circ} \mathrm{C}$ for $72 \mathrm{~h}$. Ce., Cellulose membrane; $\mathrm{Cu}$., cucumber cotyledons; H.s.Cu., cucumber cotyledons subjected to heat treatment at $50^{\circ} \mathrm{C}$ for $30 \mathrm{~s}$. Scale bars $=10 \mu \mathrm{m}$. Ap, Appressoria; Ih, Infection hyphae stained with aniline blue. B, Conidial suspensions of the wildtype 104-T and cokel1 mutant were incubated on cellophane membranes at $24^{\circ} \mathrm{C}$ for $48 \mathrm{~h}$ and the membranes were stained with $\mathrm{ZnCl} / \mathrm{KI} / \mathrm{I}_{2}$ solution. Ap, Appressoria; Ha, Halo. Scale bar $=10 \mu \mathrm{m}$. C, Percentage of appressoria forming infection hyphae. In total, at least 200 appressoria were observed for each sample, based on examination of three replicates. Means and standard errors were calculated from three independent experiments. 
In these cells, GFP-Cokel1p signals were detected on the spindle microtubules labeled by mRFP1- $\alpha$ TUB1 (Fig. 6B g through i). On the other hand, the localization of GFP-Cokellp at specific intracellular sites was not observed during appressorium formation (data not shown).

cokel1-cokel2 double mutants show an additive phenotype.

The above results indicated that CoKEL1 is involved in appressorium morphogenesis. Recently, we isolated another kelch repeat encoding gene, CoKEL2, in C. orbiculare. CoKEL2 was also involved in appressorium morphogenesis but, unlike CoKEL1, was not essential for fungal pathogenicity (Sakaguchi et al. 2008). To further investigate the roles of CoKELl and CoKEL2 in appressorium development, cokel1-cokel2 double mutants were isolated. The gene replacement vector pBIG3CKEL2Tn was introduced into the cokell mutant RK1-

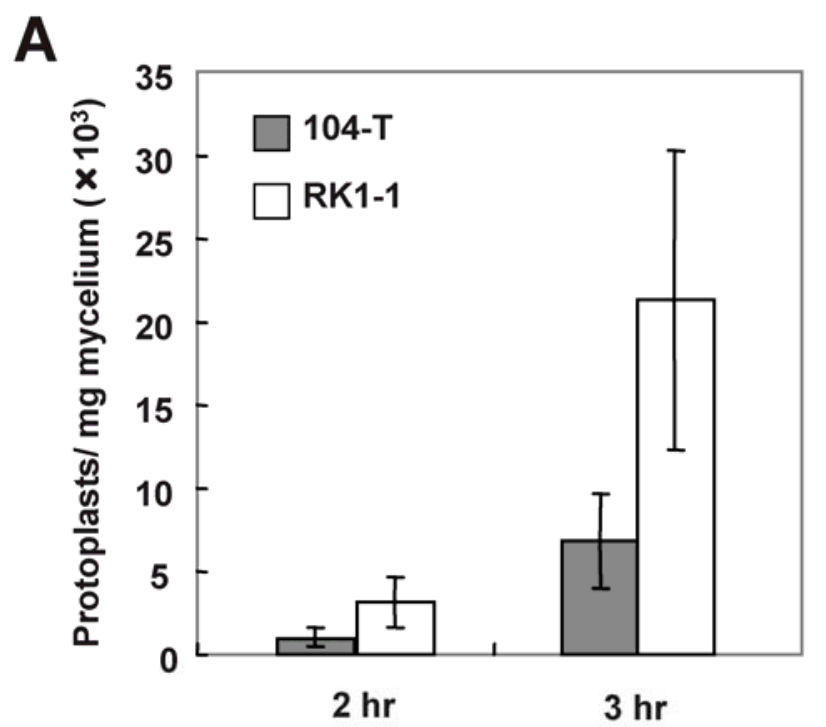

B

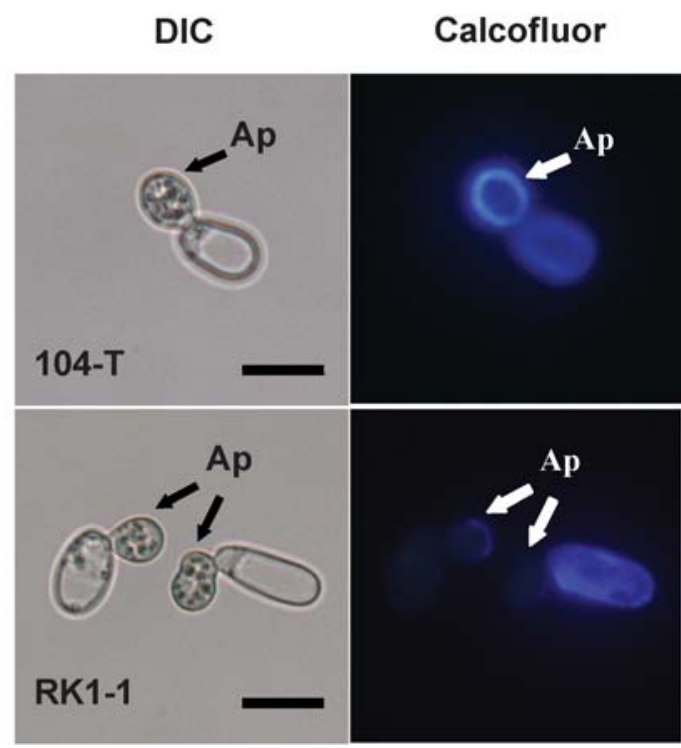

Fig. 5. Cell wall synthesis is altered in the cokell mutant. A, Sensitivity of the cokell mutant to cell-wall-degrading enzymes. Bars represent the average number of protoplasts released from wild-type or cokell mutant mycelia after incubation with Lysing enzyme for 2 or $3 \mathrm{~h}$. Means and standard deviations were calculated from five independent experiments. B, Appressoria of the cokell mutant stained with Calcofluor white to detect cell wall polysaccharides. Conidia germinated for $6 \mathrm{~h}$ were stained with Calcofluor white $\left(40 \mathrm{ng} \mathrm{ml}^{-1}\right)$. Ap, Appressoria. Scale bars $=10 \mu \mathrm{m}$.
1 by AtMT, and the replacement of CoKEL2 was confirmed by DNA gel blot analysis (data not shown). The cokell-cokel2 double mutants showed an additive phenotype for both appressorium morphogenesis and pathogenicity compared with the cokell and cokel2 single mutants. The cokell-cokel2 mutants formed abnormal appressorium similar to those of cokel2 on glass slides (Fig. 7A). Approximately $90 \%$ of cokel2 appressoria showed lateral germination, as reported previously (Sakaguchi et al. 2008), and appressoria of the cokell-cokel2 double mutant underwent lateral germination to a similar extent (Fig. 7B). On the other hand, pathogenicity of the cokellcokel2 mutants on cucumber cotyledons was reduced to the same extent as the cokell single mutant, whereas the cokel2 single mutant formed much larger necrotic lesions, similar to those of the wild type (Fig. 7C).

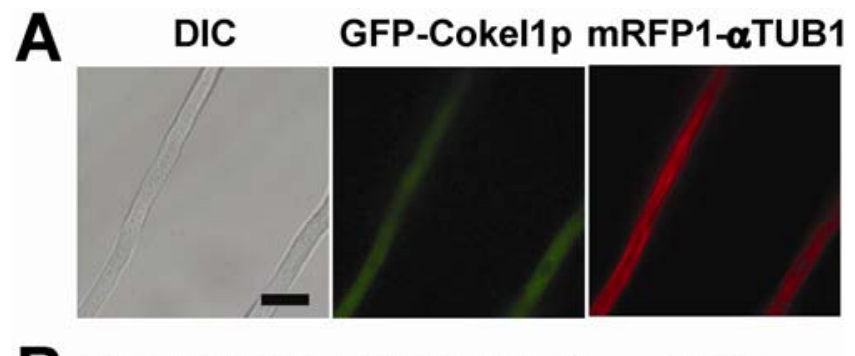

\section{B GFP-Cokel1p mRFP1- $\alpha$ TUB1 Merge}

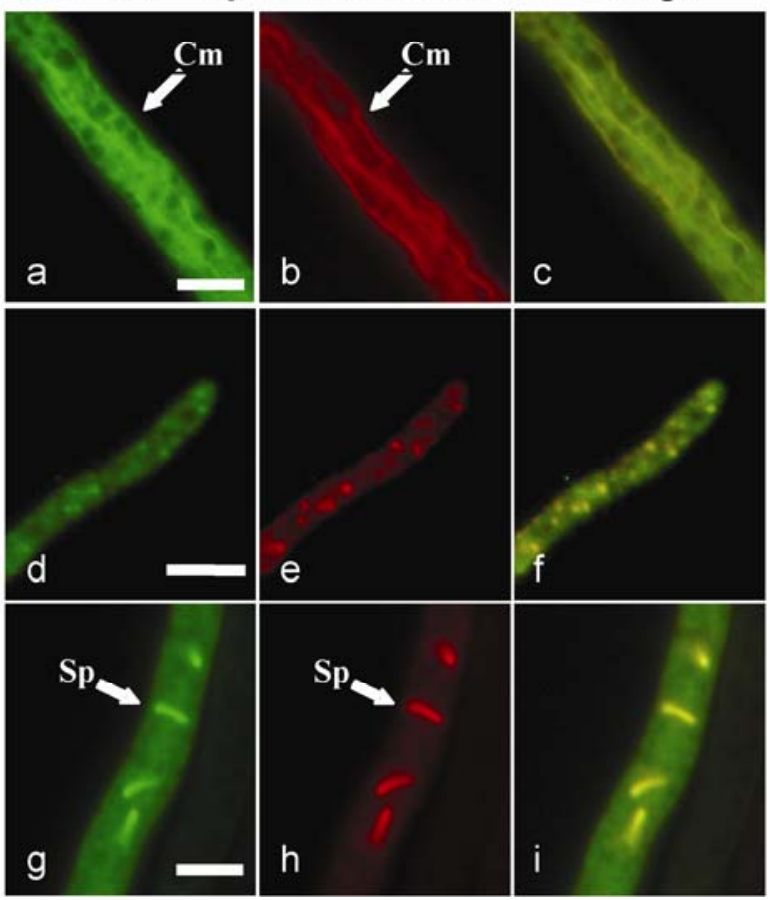

Fig. 6. Cokellp is associated with microtubules. A, GFP-CoKELl and $m R F P 1-\alpha T U B 1$ expression in vegetative hyphae grown on potato dextrose agar. Green fluorescent protein (GFP) fluorescence was distributed uniformly through the cytoplasm with no preferential labeling of specific cell components while mRFP1 fluorescence was detected as filamentous microtubule structures. Scale bars $=10 \mu \mathrm{m}$. B, Localization of Cokel1p-GFP signals to microtubules was demonstrated by coexpression of mRFP1- $\alpha \mathrm{TUB} 1$. a through c, Filamentous structure of Cokel1p-GFP corresponded to cortical microtubule labeling mRFP1- $\alpha$ TUB1. $\mathbf{d}$ through $\mathbf{f}$, Vegetative hyphae were treated with a microtubule depolymerizing drug, benomyl $\left(10 \mu \mathrm{g} \mathrm{ml}^{-1}\right)$. Cokel1p-GFP and mRFP1- $\alpha$ TUB1 fluorescence colocalized in punctate structures within the cytoplasm. $\mathbf{g}$ through $\mathbf{i}$, Hyphal cells undergoing mitosis, showing that Cokellp-GFP signals localize on spindle microtubules. $\mathrm{Cm}$, cortical microtubule; Sp, spindle microtubule; DIC, differential-interference contrast images; GFP-Cokel1p, epifluorescence images of GFP-Cokellp; mRFP1- $\alpha$ TUB1, epifluorescence images of mRFP1- $\alpha$ TUB1: merge, overlay of green and red fluorescence. Scale bars $=5 \mu \mathrm{m}$. 


\section{DISCUSSION}

Cokel1p of $C$. orbiculare is a microtubule-associated protein.

From a forward genetic screen for pathogenicity mutants in C. orbiculare, we have identified the gene CoKEL1, which encodes a protein with a $\mathrm{C}$-terminal region containing multiple repeats of the kelch motif. The kelch repeat forms $\beta$-propeller structure that is considered to function as a protein-protein interaction module mediating association with diverse partners (Adams et al. 2000; Prag and Adams 2003). For example, the Drosophila kelch protein is a component of ring canals which are the intercellular bridges connecting the nurse cells and the oocyte in an egg chamber and is involved in the transport of nurse cell cytoplasm to the oocyte. The kelch repeat is necessary and sufficient for ring canal localization (Prag and Adams 1993; Robinson and Cooley 1997). Keap1, a mammalian actin-binding protein, sequesters the transcription factor Nrf1 and protects it against electrophilic and oxidative stress (Kang et al. 2002). Arabidopsis thaliana ACBP4 and ACBP5, acylCoA-binding proteins, are involved in maintaining an oleoylCoA pool in the cytosol and transfer of oleoyl-CoA esters from

\section{A}

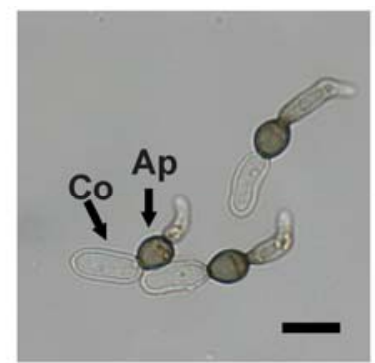

RK2-1
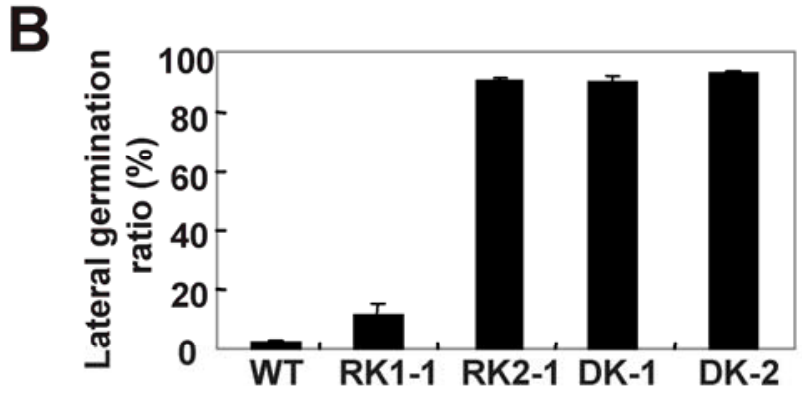

C

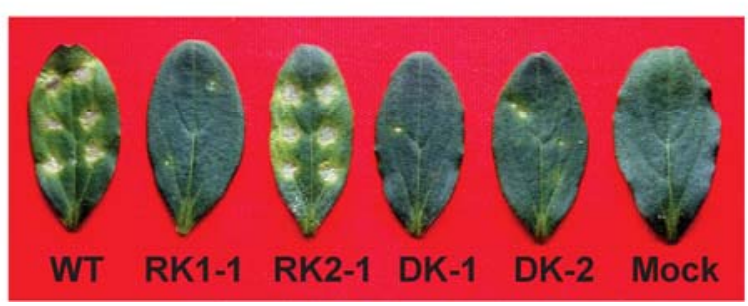

Fig. 7. Phenotype of cokell-cokel2 double mutants. A, Appressorium formation by the cokel1-cokel2 double mutant (DK-1) compared with that of cokel2 single mutant (RK2-1). Conidial suspensions of each strain in distilled water were incubated on multi-well glass slides at $24^{\circ} \mathrm{C}$ for $24 \mathrm{~h}$. Ap, appressoria; Co, conidia. Scale bars $=10 \mu \mathrm{m}$. B, Percentage of lateral germination from appressoria formed on slide glass. WT, wild-type 104-T; RK1-1, cokel1 mutant; RK2-1, cokel2 mutant; DK-1 and DK-2, cokel1cokel 2 mutants. In total, at least 200 appressoria were observed for each sample, based on examination of three replicates. Means and standard errors were calculated from three independent experiments. C, Pathogenicity of cokel1-cokel2 double mutants on intact leaves. Conidial suspensions were droplet-inoculated onto detached cucumber cotyledons and incubated at $24^{\circ} \mathrm{C}$ for 6 days. WT, wild-type 104-T; RK1-1, cokell mutant; RK2-1, cokel2 mutant; DK-1 and DK-2, cokel1-cokel2 mutants; Mock, distilled water control. the chloroplast to the endoplasmic reticulum (Leung et al. 2004). The crystal structure of a fungal galactose oxidase from Dactylium dendroides has been determined and the propeller structure shown to be the catalytic domain of the enzyme (Ito et al. 1994).

Some kelch repeat proteins are associated with the actin or microtubule cytoskeleton (Adams et al. 2000). For example, the kelch repeat in tea1p of Schizosaccharomyces pombe mediates interaction with CLIP170 tip1p, which is essential for appropriate localization at microtubule plus ends and cell apices (Brunner and Nurse 2000; Feierbach et al. 2004; Martin et al. 2005). Similarly, tea1p homologues such as Cokel2p of $C$. orbiculare and TeaA of Aspergillus nidulans associate with microtubule plus ends in growing cells (Mata and Nurse 1997; Sakaguchi et al. 2008; Takeshita et al. 2008). In the present study, we observed the localization of GFP-Cokel1p expressed from a strong constitutive promoter, because the expression level provided by the CoKEL1 native promoter was low. Significantly, GFP-Cokel1p localized at cytoplasmic and spindle microtubules, indicating that Cokl1p is also a microtubuleassociated protein. However, the cellular localization differed from that of Cokel $2 p$ in that Cokel1p was distributed along the length of microtubule filaments rather than at microtubule ends and cell apices. This finding suggests that Cokel1p is not conveyed along microtubules like tea1p or Cokel $2 p$ but, rather, resides at microtubules. Phylogenetic analysis indicated that CoKEL1 and CoKEL2 belong to different clades, which supports the idea that CoKEL1 and CoKEL2 are functionally distinct proteins.

The association of GFP-Cokel1p to microtubules was not visible in all hyphal cells and appeared to be restricted to a subset of cells. This localization pattern suggests that the association of Cokel1p with microtubules may be dependent on cell-autonomous factors, such as cell-cycle stage, and may be regulated by Cokel1p-associated proteins or signaling pathways that activate Cokel1p under specific cellular conditions or developmental stages.

The two kelch repeat proteins Cokel1p and Cokel2p are both involved in appressorium development and are microtubuleassociating proteins. Therefore, to investigate the functional relationship between Cokel1p and Cokel2p during appressorium development, we generated double mutants. The cokell-cokel2 mutants showed an additive phenotype compared with that of the single mutants, suggesting that these two kelch repeat proteins do not function in the same cellular processes.

\section{CoKEL1 is required for full virulence in C. orbiculare.}

In the human pathogen Candida albicans, a kelch repeat protein was shown to be involved in the host-fungus interaction. Thus, the MDS3 gene was required for the expression of filamentation-associated genes in alkaline media and contributed to full virulence (Davis et al. 2002). In contrast, kelch repeat proteins were not previously reported to play a role in the virulence of plant-pathogenic fungi.

Targeted disruption of CoKEL1 strongly reduced appressorium-mediated infection of host epidermal cells. Accordingly, the cokell mutants produced smaller lesions than the wild type on intact cucumber leaves. However, appressoria of cokell mutants could penetrate and form hyphae inside cellophane membranes with efficiency similar to that of the wild-type strain, indicating that CoKEL1 is not essential for differentiation of the appressorial penetration peg and infection hyphae. On the other hand, the appressoria of cokell mutants formed very few infection hyphae on intact host leaves. In C. orbiculare, the ability of some pathogenicity mutants to penetrate cucumber cotyledons can be restored by impairing host resistance with a heat-shock treatment (Asakura et al. 2006; Takano 
et al. 2006; Tanaka et al. 2007). However, heat treatment did not restore the penetration efficiency of cokell mutants on cucumber cotyledons, suggesting that the reduced pathogenicity of cokell mutants results from impaired appressorium function.

\section{CoKEL1 is involved in infection-related morphogenesis.}

Although the frequency of appressorium formation and melanization were not affected by disruption of CoKEL1, appressorium morphogenesis was perturbed, and approximately half the conidia of cokell mutants formed irregularly shaped appressoria. Moreover, vegetative hyphae of the cokell mutant were more sensitive to cell-wall-degrading enzymes than the wild type, and appressorial cell walls of the cokell mutant showed reduced staining by the chitin-specific dye Calcofluor white. These results indicate that the disruption of CoKEL1 may affect development of the appressorial cell wall. Furthermore, cytorrhysis assays showed that, even in cokell appressoria retaining normal (spherical) shape, turgor generation was significantly lower than in wild-type appressoria. These results indicate that CoKEL1 plays a role not only in appressorium morphogenesis but also in the generation of physical force by appressoria.

The generation of high turgor pressure allows the melanized appressoria of pathogens such as Colletotrichum and Magnaporthe spp. to breach the host cuticle and epidermal cell wall by means of mechanical force (Wang et al. 2005). There is evidence that melanization is critical for turgor generation by reducing the porosity of appressorial cell walls and, thus, trapping osmotically active solutes such as glycerol within the appressorial cytoplasm (Howard et al. 1991). We propose that abnormal cell wall construction in the cokell mutant allows solutes to escape from the appressorium, resulting in the generation of insufficient turgor pressure for successful penetration of host epidermal cells.

\section{MATERIALS AND METHODS}

\section{Strains and culture conditions.}

Strain 104-T (MAFF240422) of C. orbiculare (Berk. \& Mont.) Arx (syn. C. lagenarium (Pass.) Ellis \& Halst.) was used as the wild-type strain. All $C$. orbiculare strains were maintained at $24^{\circ} \mathrm{C}$ in the dark on potato dextrose media $(3.9 \%$ [wt/vol] PDA; Difco Laboratories, Detroit) or SD medium $(0.67 \%$ [wt $/ \mathrm{vol}$ ] yeast nitrogen base without amino acids, $2 \%$ [wt/vol] glucose, 2\% [wt/vol] agar). Escherichia coli DH5- $\alpha$ was used as a host for gene manipulation and was maintained on Luria-Bertani medium (Sambrook et al. 1989) at $37^{\circ} \mathrm{C}$. Agrobacterium tumefaciens $\mathrm{C} 58 \mathrm{C} 1$ was used as a T-DNA donor for fungal transformation and was maintained on AB-minimal medium (Lichtenstein and Draper 1986) at $28^{\circ} \mathrm{C}$.

\section{Fungal transformation.}

Protoplast preparation and transformation of $C$. orbiculare were performed according to the method described previously (Kubo et al. 1991). In REMI mutagenesis, the vector pCB1490 containing the hygromycin resistance gene was introduced into fungal protoplasts with restriction enzyme PstI. Transformants with the hygromycin resistance gene were selected on regeneration medium containing hygromycin B (Wako Chemicals, Osaka, Japan) at $50 \mu \mathrm{g} / \mathrm{ml}$. Strain P24 was isolated after screening the REMI transformants. C. orbiculare was transformed by AtMT as described previously (Tsuji et al. 2003a). Hygromycinresistant transformants were selected on PDA medium containing hygromycin B (Wako Chemicals) at $100 \mu \mathrm{g} / \mathrm{ml}$, cefotaxim (Wako Chemicals) at $100 \mu \mathrm{g} / \mathrm{ml}$, and spectinomycin (Wako Chemicals) at $100 \mu \mathrm{g} / \mathrm{ml}$. Bialaphos-resistant transformants were selected on SD medium containing bialaphos at $4 \mu \mathrm{g} / \mathrm{ml}$
(Meiji Seika Kaisha, Ltd., Tokyo), cefotaxim at $100 \mu \mathrm{g} / \mathrm{ml}$, and spectinomycin at $100 \mu \mathrm{g} / \mathrm{ml}$. Sulfonylurea-resistant transformants were selected on SD medium containing chlorimuron ethyl (Chem Service, West Chester, PA, U.S.A.) at $4 \mu \mathrm{g} / \mathrm{ml}$, cefotaxim at $100 \mu \mathrm{g} / \mathrm{ml}$, and spectinomycin at $100 \mu \mathrm{g} / \mathrm{ml}$.

\section{Cloning and sequencing.}

To identify the disrupted gene in mutant P24, a TAIL-PCR protocol was applied to genomic DNA (Liu et al. 1995). Fungal genomic DNA flanking the pCB1490 plasmid insert was analyzed as described previously (Tsuji et al. 2003a). Amplified PCR products were sequenced with the Big-Dye terminator cycle sequencing ready reaction kit (Applied Biosystems Japan, Ltd., Tokyo) and an ABI PRISM 310 automated DNA sequencer (Applied Biosystems Japan, Ltd.). Genomic clones of CoKEL1 were selected from a cosmid library of C. orbiculare 104-T by PCR using primer pair P24S1 (5'-TAGACCCA CACGTCGTTGAA-3') and P24AS1 (5'-ACGTGAGCGACA TGAACAAG-3') designed from the obtained DNA sequence. To obtain the entire CoKELl ORF, the EZ::TN <KAN-2> Insertion Kit (Epicenter, Madison, WI, U.S.A.) was used to insert a transposon containing a selection marker into the target DNA. Clones containing the $<\mathrm{KAN}-2>$ transposon was sequenced using primers KAN2-RP2 (5'-GGAAGATCTTTG TGCAATGTAACATCAGAG-3') and KAN2-FP3 (5'-GCAG GCGATGCAAGCTTCAGGGTTG-3'). RACE analysis was performed using the 5' Full RACE Core Set (Takara Bio, Ohtsu, Japan) to determine the transcription initiation site of CoKEL1. The CoKEL1sequence is available in the DDBJ database under accession no. AB259752.

\section{Plasmid constructs.}

To construct the CoKEL1 gene-replacement vector p24cosKEL1Tn, the 2.7-kb transposon EZ::TN <KAN-2>, in which the kanamycin resistance gene was replaced by the ampicillin resistance gene and the hygromycin resistance gene, was inserted into $A$. tumefaciens binary cosmid vector p24cosKEL1 containing the CoKEL1 gene. The vector p24cosKEL1Tn was selected from randomly inserted clones and contained the transposon at $320 \mathrm{bp}$ downstream from the start codon. Disruption of the CoKELl gene was confirmed by genomic DNA blot analysis of selected transformants.

For construction of the EGFP::CoKEL1 fusion gene regulated by a constitutive promoter, A. tumefaciens binary vector pBI-TEFpGFPB was used as starting construct. The plasmid pBI-TEFpGFPB contains the bialaphos resistance gene and the enhanced GFP (EGFP) ORF linked by replacement of the stop codon with a spacer encoding five glycine residues. The expression of $E G F P$ was controlled by the translation elongation factor from Aureobasidium pullulans derived from pTEFEGFP (van den Wymelenberg et al. 1997). The full region of the CoKEL1 ORF was amplified by PCR with primers KEL1S2 (5'-CGGAATTCATGGCTGATTCGGCCAGGT-3') and KEL1AS3 (5'-CGGGATCCTAGACCTCGATGGTGAAGT$3^{\prime}$ ) and was digested with EcoRI and BamHI, introduced into the EcoRI and BamHI site of pBI-TEFpGFPB, and designated as pBI-TEFpGFP-KEL1.

For construction of the EGFP::CoKEL1 fusion gene regulated by the native CoKEL1 promoter, Agrobacterium tumefaciens binary vector pBI-SCD1pGFPB was used as starting construct. The plasmid pBI-SCD1pGFPB contains the bialaphos resistance gene and EGFP ORF linked by replacement of the stop codon with a spacer encoding five glycine residues. The EGFP was controlled by a 221-bp 5' upstream region of the $C$. orbiculare SCD1 gene (Takano et al. 2001). The last codon of EGFP was fused to 15 nucleotides encoding a glycine linker followed by the EcoRI, BamHI site and the termi- 
nator of the glucoamylase gene of Aspergillus awamori. To construct the EGFP::CoKEL1 fusion vector, the full region of the CoKEL1 ORF was amplified by PCR with primers KEL1S2 and KEL1AS3 and digested with EcoRI and BamHI, introduced into the EcoRI and BamHI site of pBISCD1pGFPB, and designated as pBI-SCD1pGFP-KEL1. The $S C D 1$ promoter was then exchanged for the native CoKEL1 promoter. However, use of the CoKEL1 promoter containing its $\mathrm{UORF}$ would have introduced a premature stop codon upstream of the start ATG of EGFP. To avoid this, the SCD1 promoter and EGFP containing a Kozack sequence was exchanged for EGFP without a Kozack sequence upstream of the ATG codon, which was amplified by PCR using primers GFPATG (5'-GATTCTAGAATGGTGAGCAAGGGCGAG$\left.3^{\prime}\right)$ and eGFPASgly (5'-CGGAATTCACCACCACCACCACC CTTGTACAGCTCGTCCAT-3'). The GFPATG primer contains a terminal $X b a I$ site, whereas eGFPASgly contains a terminal EcoRI site. The amplified product was digested with $X b a \mathrm{I}$ and $\mathrm{BamHI}$ and introduced into the $\mathrm{Xba \textrm {I }}$ and BamHI site of pBI-SCD1pGFP::KEL1, which contained the SCD1 promoter and EGFP. The resulting plasmid was designated pBIATGGFP::KEL1. The 1,083-bp 5' upstream region of the CoKEL1 gene was amplified by PCR using primers KEL1plusAS (5'-GGACTAGTATCAGCCATGGTTCGGCG$\left.3^{\prime}\right)$, containing uORF sequence overlapping CoKEL1 (ATGGCTGAT) and a terminal SpeI, and T7 (5'TAATACGACTCACTATAGGG-3'). The plasmid pBSKEL1, containing a 1,083-bp 5' upstream region of the CoKEL1 gene and the CoKEL1 ORF, was used as a template for PCR. The amplified product was digested with SpeI and introduced into the $\mathrm{XbaI}$ site of pBI-ATGGFP::KEL1. The resulting clone was named pBI-GFP::KEL1.

For labeling of the microtubule, pBI-SCD1pmRFP1:: $\alpha$ TUB1S was used as previously described (Sakaguchi et al. 2008).

\section{Genomic DNA blot analysis.}

Genomic DNA of $C$. orbiculare was isolated from mycelia and DNA blot analysis was performed as described previously (Takano et al. 1997). DNA digestion, gel electrophoresis, labeling of probes, and hybridization were performed according to the manufacturer's instructions and standard methods (Sambrook et al. 1989). DNA probes were labeled with digoxigenin (DIG)-dUTP using the BcaBEST DIG labeling kit (Takara Bio). Hybridized DNA was detected by Anti-Digoxygenin-AP Fab fragments (Roche Diagnostics, Tokyo) and light emission generated by enzymatic dephosphorylation of CDP-Star Detection Reagent (GE Healthcare, Tokyo) by alkaline phosphatase was detected using FUJIFILM LAS1000 plus.

\section{Pathogenicity tests.}

Inoculation assays on cucumber cotyledons (Cucumis sativus L. 'Suyo') were performed as described previously (Tsuji et al. 2003b). For estimation of invasive growth ability, 3- $\mu$ l drops of conidial suspension $\left(5 \times 10^{5}\right.$ conidia $\left.\mathrm{ml}^{-1}\right)$ were spotted onto wound sites prepared by scratching the leaf surface with a sterile toothpick. After inoculation, the cotyledons were incubated in a humid box at $24^{\circ} \mathrm{C}$ with a 16 -h photoperiod for 5 days.

\section{Microscopy.}

For observation of appressorium formation and penetration in vitro, conidia were harvested from 7-day-old PDA cultures and suspended in distilled water. The conidial suspension was adjusted to $10^{5}$ conidia $\mathrm{ml}^{-1}$ and 20 - $\mu$ ldroplets were placed onto eight-well multitest glass slides (ICN Biomedicals, Aurora, OH, U.S.A.) or cellophane membranes (Wako Chemi- cals) and incubated at $24^{\circ} \mathrm{C}$ in the dark. Cellulose dissolution was assessed by staining the membranes with a solution containing $\mathrm{ZnCl}_{2}\left(50 \%\right.$, wt/vol), $\mathrm{KI}\left(20 \%\right.$, wt/vol), and $\mathrm{I}_{2}(0.5 \%$, wt/vol). Germlings were observed with a Nikon ECLIPSE E600 microscope using differential-interference contrast optics (Nikon, Tokyo). For observation of infectious hypha in cucumber leaves, the conidial suspension of each strain was spotted onto the abaxial surface of cucumber cotyledons and incubated at $24^{\circ} \mathrm{C}$ for 3 days. Then, the inoculation site was peeled off and stained with $0.1 \%$ lactophenol-anilin blue. For observation of GFP and mRFP1 fluorescence, cells were viewed with an epifluorescence microscope equipped with a GFP(R)-BP filter (460- to 500-nm excitation filter, 505-nm dichroic mirror, and 510 - to $560-\mathrm{nm}$ barrier filter) and G-2A filter (510- to $560-\mathrm{nm}$ excitation filter, 575-nm dichroic mirror, and $>590-\mathrm{nm}$ barrier filter), respectively. For staining cell wall polysaccharides, Fluorescent Brightener 28 (Calcofluor white, MP Biomedicals, Inc., Burlingame, CA, U.S.A.) (40 $\mathrm{ng} \mathrm{m}^{-1}$ ) was applied to germlings grown for $6 \mathrm{~h}$ on multitest glass slides. After 10 min, the stained cells were washed with distilled water and observed by epifluorescence microscopy with a UV-1A filter (365/10-nm excitation filter, 400-nm dichroic mirror, and 400 $\mathrm{nm}$-barrier filter).

For protoplast preparation, conidia of each strain were incubated in potato-sucrose liquid medium at $24^{\circ} \mathrm{C}$ for 3 days with shaking at $90 \mathrm{rpm}$. Mycelia were collected by filtration, washed in a solution of $1.2 \mathrm{M} \mathrm{MgSO}_{4}$ and $10 \mathrm{mM} \mathrm{Na} \mathrm{HPO}_{4}$, and then transferred to the same solution supplemented with Lysing enzymes $(5 \mathrm{mg} / \mathrm{ml})$ (Sigma), and incubated at $24^{\circ} \mathrm{C}$. Protoplasts released from mycelia after treatment with enzymes for 2 and $3 \mathrm{~h}$ were then counted on a hemacytometer slide using a microscope.

\section{ACKNOWLEDGMENTS}

We thank R. O'Connell for carefully reading the manuscript and giving useful suggestions. This work was supported by Grants-in-Aid for Scientific Research from the Ministry of Education, Culture, Sports, Science and Technology (nos.19380029 and 21380031).

\section{LITERATURE CITED}

Adams, J., Kelso, R., and Cooley, L. 2000. The kelch repeat superfamily of proteins: Propellers of cell function. Trends Cell Biol. 10:17-24.

Arellano, M., Niccoli, T., and Nurse, P. 2002. Tea3p is a cell end marker activating polarized growth in Schizosaccharomyces pombe. Curr. Biol. 12:751-756.

Asakura, M., Okuno, T., and Takano, Y. 2006. Multiple contributions of peroxisomal metabolic function to fungal pathogenicity in Colletotrichum lagenarium. Appl. Environ. Microbiol. 72:6345-6354.

Asakura, M., Ninomiya, S., Sugimoto, M., Oku, M., Yamashita, S., Okuno, T., Sakai, Y., and Takano, Y. 2009. Atg26-mediated pexophagy is required for host invasion by the pla1291-nt pathogenic fungus Colletotrichum orbiculare. Plant Cell 1291-1304.

Bechinger, C., Giebel, K. F., Schnell, M., Leiderer, P., Deising, H. B., and Bastmeyer, M. 1999. Optical measurements of invasive forces exerted by appressoria of a plant pathogenic fungus. Science 285:1896-1899.

Brunner, D., and Nurse, P. 2000. CLIP170-like tip1p spatially organizes microtubular dynamics in fission yeast. Cell 102:695-704.

Davis, D. A., Bruno, V. M., Loza, L., Filler, S. G., and Mitchell, A. P. 2002. Candida albicans Mds3p, a conserved regulator of $\mathrm{pH}$ responses and virulence identified through insertional mutagenesis. Genetics 162:1573-1581.

Feierbach, B., Verde, F., and Chang, F. 2004. Regulation of a formin complex by the microtubule plus end protein tea1p. J. Cell Biol. 165:697707.

Fukui, Y., Miyake, S., Satoh, M., and Yamamoto, M. 1989. Characterization of the Schizosaccharomyces pombe ral2 gene implicated in activation of the ras1 gene product. Mol. Cell. Biol. 9:5617-5622.

Harashima, T., and Heitman, J. 2002. The Galpha protein Gpa2 controls yeast differentiation by interacting with kelch repeat proteins that mimic Gbeta subunits. Mol. Cell 10:163-173. 
Howard, R. J., Ferrari, M. A., Roach, D. H., and Money, N. P. 1991. Penetration of hard substrates by a fungus employing enormous turgor pressures. Proc. Natl. Acad. Sci. U.S.A. 88:11281-11284.

Ito, N., Phillips, S. E., Yadav, K. D., and Knowles, P. F. 1994. Crystal structure of a free radical enzyme, galactose oxidase. J. Mol. Biol. 238:794-814.

Kang, M. I., Kobayashi, A., Wakabayashi, N., Kim, S. G., and Yamamoto, M. 2002. Scaffolding of Keap1 to the actin cytoskeleton controls the function of Nrf2 as key regulator of cytoprotective phase 2 genes. Proc. Natl. Acad. Sci. U.S.A. 101:2046-2051.

Kimura, A., Takano, Y., Furusawa, I., and Okuno, T. 2001. Peroxisomal metabolic function is required for appressorium-mediated plant infection by Colletotrichum lagenarium. Plant Cell 13:1945-1957.

Kojima, K., Kikuchi, T., Takano, Y., Oshiro, T., and Okuno, T. 2002. The mitogen-activated protein kinase gene MAF1 is essential for the early differentiation phase of appressorium formation in Colletotrichum lagenarium. Mol. Plant-Microbe Interact. 15:1268-1276.

Kubo, Y., and Furusawa, I. 1991. Melanin biosynthesis: Prerequisite for successful invasion of the plant host by appressoria of Colletotrichum and Pyricularia. Pages 205-217 in: The Fungal Spore and Disease Initiation in Plants and Animals. G. T. Cole and H. C. Hoch, eds. Plenum Publishing, New York.

Kubo, Y., Nakamura, H., Kobayashi, K., Okuno, T., and Furusawa, I. 1991. Cloning of a melanin biosynthetic gene essential for appressorial penetration of Colletotrichum lagenarium. Mol. Plant-Microbe Interact. 4:440-445.

Kubo, Y., Takano, Y., Endo, N., Yasuda, N., Tajima, S., and Furusawa, I. 1996. Cloning and structural analysis of the melanin biosynthesis gene SCD1 encoding scytalone dehydratase in Colletotrichum lagenarium. Appl. Environ. Microbiol. 62:4340-4344.

Lee, K. S, Irie, K., Gotoh, Y., Watanabe, Y., Araki, H., Nishida, E., Matsumoto, K., and Levin D. E. 1993. A yeast mitogen-activated protein kinase homolog (Mpk1p) mediates signalling by protein kinase C. Mol. Cell. Biol. 13:3067-3075.

Leung K. C., Li, H. Y., Mishra, G., and Chye, M. L. 2004. ACBP4 and ACBP5, novel Arabidopsis acyl-CoA-binding proteins with kelch motifs that bind oleoyl-CoA. Plant Mol. Biol. 55:297-309.

Lichtenstein, C., and Draper, J. 1986. Genetic engineering of plants. Pages 67-119 in: DNA Cloning: A Practical Approach, Vol. 2. D. M. Glover, ed. IRL Press, Oxford.

Liu, Y. G., Mitsukawa, N., Teruo, O., and Whittier, R. F. 1995. Efficient isolation and mapping of Arabidopsis thaliana T-DNA insert junctions by thermal asymmetric interlaced PCR. Plant J. 8:457-463.

Martin, S. G., McDonald, W. H., Yates, J. R, and Chang, F. 2005. Tea4p links microtubule plus ends with the formin for $3 p$ in the establishment of cell polarity. Dev. Cell. 8:479-491.

Mata, J., and Nurse, P. 1997. tea1 and the microtubular cytoskeleton are important for generating global spatial order within the fission yeast cell. Cell 89:939-949.

Peeters, T, Versele, M., and Thevelein, J. M. 2007. Directly from Galpha to protein kinase A: The kelch repeat protein bypass of adenylate cyclase. Trends Biochem. Sci. 32:547-554.

Perpetua, N. S., Kubo, Y., Yasuda, N., Takano, Y., and Furusawa, I. 1996. Cloning and characterization of a melanin biosynthetic THR1 reductase gene essential for appressorial penetration of Colletotrichum lagenarium. Mol. Plant-Microbe Interact. 9:323-329.

Philips, J., and Herskowitz, I. 1998. Identification of Kel1p, a kelch domain-containing protein involved in cell fusion and morphology in Saccharomyces cerevisiae. J. Cell Biol. 143:375-389.

Prag, S., and Adams, J. C. 1993. kelch encodes a component of intercellular bridges in Drosophila egg chambers. Cell 72:681-693.

Prag, S., and Adams, J. C. 2003. Molecular phylogeny of the kelch-repeat superfamily reveals an expansion of BTB/kelch proteins in animals. BMC Bioinf. 17:42

Robinson, D. N., and Cooley, L. 1997. Drosophila kelch is an oligomeric ring canal actin organizer. J. Cell Biol. 138:799-810.

Sambrook, J., E. F. Fritsch, and T. Maniatis. 1989. Molecular Cloning: A
Laboratory Manual. Cold Spring Harbor Laboratory Press, Cold Spring Harbor, NY, U.S.A.

Sakaguchi, A., Miyaji, T., Tsuji, G., and Kubo, Y. 2008. Kelch repeat protein Clakel2p and calcium signaling control appressorium development in Colletotrichum lagenarium. Eukaryot. Cell. 7:102-111.

Takano, Y., Kubo, Y., Shimizu, K., Mise, K., Okuno, T., and Furusawa, I. 1995. Structural analysis of PKS1, a polyketide synthase gene involved in melanin biosynthesis in Colletotrichum lagenarium. Mol. Gen. Genet. 249:162-167.

Takano, Y., Kubo, Y., Kawamura, C., Tsuge, T., and Furusawa, I. 1997. The Alternaria alternata melanin biosynthesis gene restores appressorial melanization and penetration of cellulose membranes in the melanindeficient albino mutant of Colletotrichum lagenarium. Fungal Genet. Biol. 21:131-140.

Takano, Y., Kikuchi, T., Kubo, Y., Hamer, J. E., Mise, K., and Furusawa, I. 2000. The Colletotrichum lagenarium MAP kinase gene CMK1 regulates diverse aspects of fungal pathogenesis. Mol. Plant-Microbe Interact. 13:374-383.

Takano, Y., Oshiro, E., and Okuno. T, 2001. Microtubule dynamics during infection-related morphogenesis of Colletotrichum lagenarium. Fungal Genet. Biol. 34:107-121.

Takano, Y., Takayanagi, N., Hori, H., Ikeuchi, Y., Suzuki, T., Kimura, A., and Okuno, T. 2006. A gene involved in modifying transfer RNA is required for fungal pathogenicity and stress tolerance of Colletotrichum lagenarium. Mol. Microbiol. 60:81-92.

Takeshita, N., Higashitsuji, Y., Konzack, S., and Fischer, R. 2008. Apical sterol-rich membranes are essential for localizing cell end markers that determine growth directionality in the filamentous fungus Aspergillus nidulans. Mol. Biol. Cell 19:339-3351.

Tamura, K., Dudley, J., Nei, M., and Kumar, S. 2007. MEGA4: Molecular Evolutionary Genetics Analysis (MEGA) software version 4.0. Mol. Biol. Evol. 24:1596-1599.

Tanaka, S., Yamada, K., Yabumoto, K., Fujii, S., Huser, A., Tsuji, G. Koga, H., Dohi, K., Mori, M., Shiraishi, T., O’Connell, R., and Kubo Y. 2007. Saccharomyces cerevisiae SSD1 orthologues are essential for host infection by the ascomycete plant pathogens Colletotrichum lagenarium and Magnaporthe grisea. Mol. Microbiol. 64:1332-1349.

Tsuji, G., Kenmochi, Y., Takano, Y., Sweigard, J., Farrall, L., Furusawa, I., Horino, O., and Kubo, Y. 2000. Novel fungal transcriptional activators, Cmr1p of Colletotrichum lagenarium and Pig1p of Magnaporthe grisea, contain Cys2His2 zinc finger and Zn (II)2Cys6 binuclear cluster DNA binding motifs, and regulate transcription of melanin biosynthesis genes in a developmentally specific manner. Mol. Microbiol. 38:940954.

Tsuji, G., Fujii, S., Fujihara, N., Hirose, C., Tsuge, S., Shiraishi, T., and Kubo, Y. 2003a. Agrobacterium tumefaciens-mediated transformation for random insertional mutagenesis in Colletotrichum lagenarium. J. Gen. Plant Pathol. 69:230-239.

Tsuji, G., Fujii, S., Tsuge, S., Shiraishi, T., and Kubo, Y. 2003b. The Colletotrichum lagenarium Ste12-like gene CST1 is essential for appressorium penetration. Mol. Plant-Microbe Interact. 16:315-325.

van den Wymelenberg A. J., Cullen, D., Spear, R. N., Schoenike, B., and Andrews, J. H. 1997. Expression of green fluorescent protein in Aureobasidium pullulans and quantification of the fungus on leaf surfaces. Biotechniques 23:686-690.

Wang, Z. Y., Jenkinson, J. M., Holcombe, L. J., Soanes, D. M., VeneaultFourrey, C., Bhambra, G. K., and Talbot, N. J. 2005. The molecular biology of appressorium turgor generation by the rice blast fungus Magnaporthe grisea. Biochem. Soc. Trans. 33:384-388.

Xu, J. R., Staiger, C. J., and Hamer, J. E. 1998. Inactivation of the mitogen-activated protein kinase Mps1 from the rice blast fungus prevents penetration of host cells but allows activation of plant defense responses. Proc. Natl. Acad. Sci. U.S.A. 95:12713-12718.

Yamauchi, J., Takayanagi, N., Komeda, K., Takano, Y., and Okuno, T. 2004. cAMP-pKA signaling regulates multiple steps of fungal infection cooperatively with Cmk1 MAP kinase in Colletotrichum lagenarium. Mol. Plant-Microbe. Interact. 17:1355-1365. 